Review began 10/26/2021 Review ended 11/08/2021 Published 11/12/2021

๑) Copyright 2021

Sebastian et al. This is an open access article distributed under the terms of the Creative Commons Attribution License CC BY 4.0., which permits unrestricted use, distribution, and reproduction in any medium, provided the original author and source are credited.

\section{Cutaneous Absidia corymbifera in a Lupus Nephritis Patient}

Kuruvilla K. Sebastian ${ }^{1}$, Husam Alzayer ${ }^{1}$, Elizabeth Abraham ${ }^{2}$, Darren Roche ${ }^{3}$, Donal Reddan ${ }^{4}$, David Lappin ${ }^{4}$

1. Department of Renal Medicine, Cork University Hospital, Cork, IRL 2. Internal Medicine, Mater Private Network, Cork, IRL 3. Department of Dermatology, University Hospital Galway, Galway, IRL 4. Department of Nephrology, University Hospital Galway, Galway, IRL

Corresponding author: Kuruvilla K. Sebastian, kevin.kallarackal@gmail.com

\section{Abstract}

A 28-year-old farmer with class IV lupus nephritis presented with a two-week history of a right shin lesion. The lesion was purple in color, fungating, and indurated with a focus of deep ulceration at the inferior pole and punctate, bleeding from its surface. Three months earlier, he was started on induction immunosuppression for a relapse of his lupus nephritis. Since the diagnosis of lupus nephritis, nine years previously, he had had six flares of his disease and had been treated at different time points with cyclophosphamide, rituximab, and high-dose corticosteroids, without adverse events. Laboratory investigations showed improving kidney function (chronic kidney disease [CKD] stage IV) with reducing proteinuria, on his current immunosuppressive regimen. The differential diagnosis for this lesion was calciphylaxis, pyoderma gangrenosum, vasculitic lesion, or an infection. Histology and microbiological analysis confirmed the presence of Absidia corymbifera. He was treated with a combination of isavuconazole, reduction of his immunosuppressive agents, excision of the lesion, and skin grafting.

Categories: Dermatology, Infectious Disease, Nephrology

Keywords: cutaneous mucormycosis, isavuconazole, lupus nephritis, absidia corymbifera, mucormycosis

\section{Introduction}

We present in this report a case of cutaneous mucormycosis with a rare opportunistic pathogen, Absidia corymbifera, in an immunosuppressed patient with class IV lupus nephritis. Our aim is to describe the natural history of its presentation, the diagnostic process and to finally discuss the complexity involved in treating this fungal infection. We also discuss the challenges faced as a consequence of immunosuppression and the side effects of the treatment in this case report.

\section{Case Presentation}

A 28-year-old farmer presented with a two-week history of a right shin lesion. The lesion was purple in color, fungating, and indurated with a focus of deep ulceration at the inferior pole and punctate bleeding from its surface (Figure 1). 


\section{Cureus}

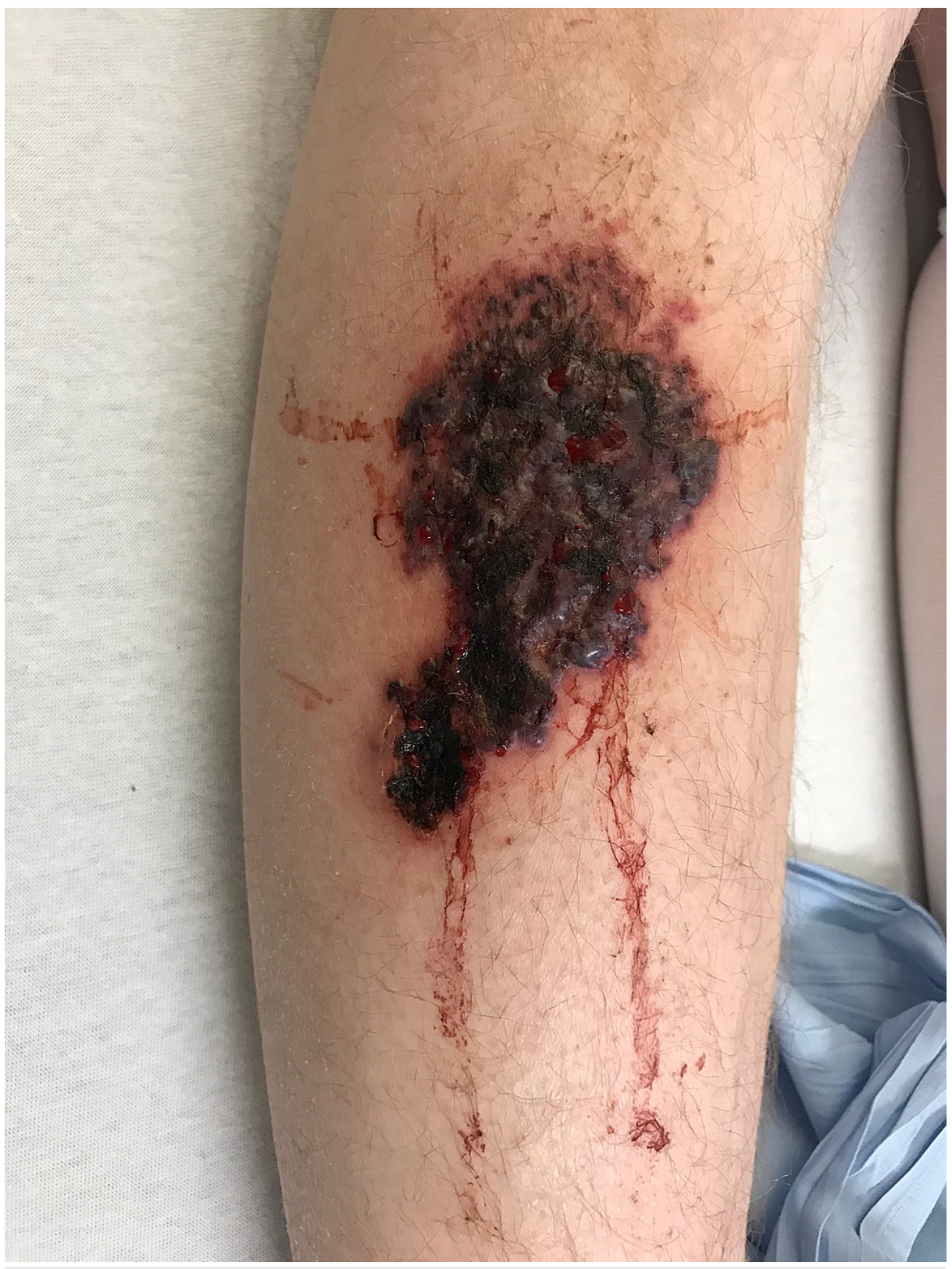

FIGURE 1: Mucormycosis lesion on the right shin.

He had been diagnosed with class IV lupus nephritis nine years ago. He had had six flares of his disease and had been treated at different time points with cyclophosphamide, rituximab, and high-dose corticosteroids, without adverse events. Three months earlier, he was started on Mycophenolate mofetil (one gram twice daily) and high-dose corticosteroids (60 mg daily) for his latest flare of lupus nephritis. His flare was characterized by a rise in creatinine from a baseline of $120 \mathrm{umol} / \mathrm{L}$ to a peak creatinine of $550 \mathrm{umol} / \mathrm{L}$ and his urine protein creatinine ratio (UPCR) was $433 \mathrm{mg} / \mathrm{mmol}$.

Initial laboratory investigations showed baseline kidney function (chronic kidney disease [CKD] stage IV) with reducing proteinuria on his current immunosuppressive regimen. Inflammatory markers were within normal limits, with no evidence of ongoing lupus activity. A plain X-ray of his right tibia/fibula did not show any abnormality, while an MRI reported soft-tissue edema. A biopsy was performed for further histopathological and microbiological analysis.

Histology of the lesion demonstrated florid acute and chronic inflammatory processes (Figure 2, Panel A), periodic acid-Schiff (PAS) stain for fungal organisms was positive for broad-based fungal hyphae with branching (Figure 2, Panel B, arrowhead) and angioinvasion (Figure 2, Panel C, long arrow). Microbiology confirmed the presence of Absidia corymbifera in the culture of the tissue sample as well as in the ulcer slough. 


\section{Cureus}
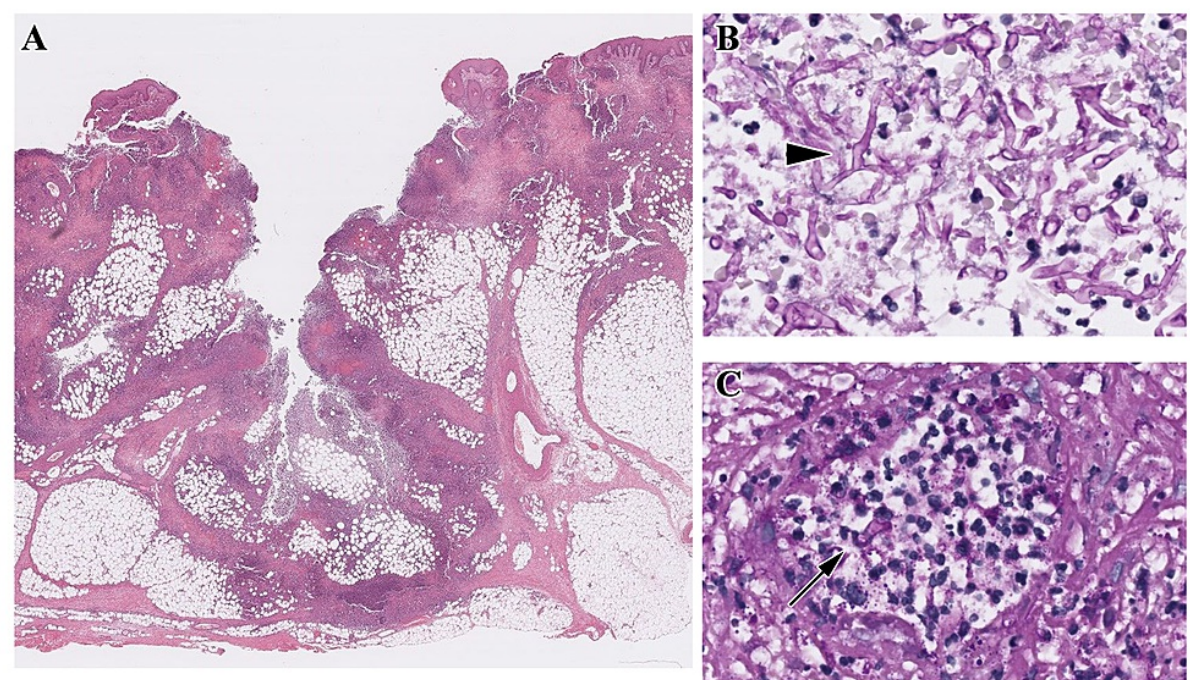

FIGURE 2: Panel A: Inflammation of the dermis, Panel B: Periodic acidSchiff (PAS) stain showing fungal hyphae (arrow-head), and Panel C: angioinvasion by fungal hyphae.

Upon diagnosis, the lesion was excised, followed by skin grafting. He was initially treated with IV liposomal amphotericin ( $3 \mathrm{mg} / \mathrm{kg}$ once daily) and a reduction of his immunosuppressive agents. Following a deterioration in his renal function, amphotericin was switched to isavuconazole (200 $\mathrm{mg}$ IV every eight hours for two days, then $200 \mathrm{mg}$ once daily orally).

His treatment was complicated by severe symptomatic anemia, which we believed was due to a combination of myelotoxicity from mycophenolate mofetil and the intravenous preparation of Isavuconazole causing pancytopenia. This particular complication was managed by reducing the mycophenolate mofetil dose and switching IV isavuconazole to an oral preparation and titrating the erythropoietin dosage. The patient also developed hypogammaglobulinemia and was treated with IV immunoglobulin for an interim. He successfully completed three months of treatment with regular monitoring. Figure 3 shows the site of the debrided lesion, three months after skin grafting. 


\section{Cureus}

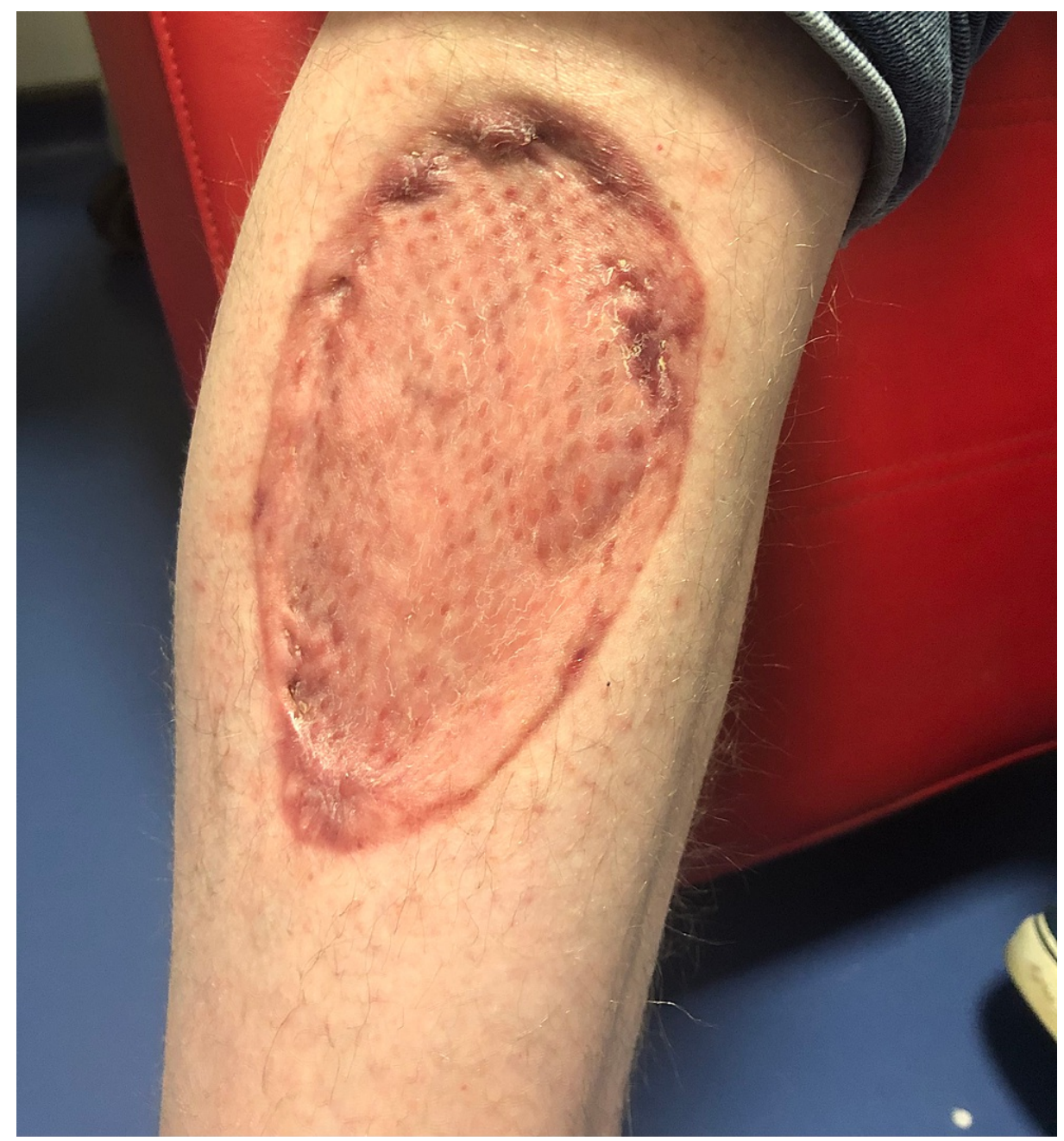

FIGURE 3: Right shin, three months after lesion debridement and skin grafting.

\section{Discussion}

Absidia corymbifera is a rare opportunistic fungus, known to cause pulmonary, CNS, rhinocerebral and cutaneous mucormycosis. Mucormycosis is an infection typically caused by fungi of the class Zygomycetes and of the order Mucorales [1]. The most common species to cause infection in humans belong to the genus Rhizopus [1].

This was a particularly difficult case with regards to diagnosis and treatment. Early multi-disciplinary team involvement was key in the successful management of this case.

The painless presentation of this lesion and stage IV CKD made the diagnosis of calciphylaxis unlikely [2]. Although biopsy of calciphylaxis lesions is the standard of diagnosis, there is a concern it could lead to new lesions and non-healing ulcers [2]. Therefore, we were reassured in proceeding with a biopsy of the lesion. The patient's occupation as a farmer and immunosuppressed status was important in considering an infective etiology or mucormycosis as a differential diagnosis for the lesion.

Balancing the immunosuppression dose with the treatment of the mucormycosis was challenging. We had initially lowered the immunosuppression doses to allow treatment of the infection. However, the patients' kidney function deteriorated as a result. But, the immunosuppression was only titrated up once the infection had been reasonably treated.

The patient developed severe symptomatic anemia (hemoglobin as low as $6.0 \mathrm{~g} / \mathrm{dL}$ ) as a side effect of the IV Isavuconazole and mycophenolate mofetil. We managed to treat this anemia without giving any blood transfusions to a potential renal transplant recipient by reducing mycophenolate mofetil and switching to oral isavuconazole. 


\section{Cureus}

\section{Conclusions}

Absidia corymbifera is a rare cause of mucormycosis. This case highlights the natural history of its transmission (i.e., through the soil in a young immunosuppressed farmer). It is exclusively an opportunistic pathogen and its cutaneous manifestation is a painless, fungating lesion as opposed to a painful lesion seen in calciphylaxis. Early multi-disciplinary team involvement, debridement, and treatment with isavuconazole were key in successfully treating this condition.

\section{Additional Information}

\section{Disclosures}

Human subjects: Consent was obtained or waived by all participants in this study. Conflicts of interest: In compliance with the ICMJE uniform disclosure form, all authors declare the following: Payment/services info: All authors have declared that no financial support was received from any organization for the submitted work. Financial relationships: All authors have declared that they have no financial relationships at present or within the previous three years with any organizations that might have an interest in the submitted work. Other relationships: All authors have declared that there are no other relationships or activities that could appear to have influenced the submitted work.

\section{Acknowledgements}

I would like to acknowledge that this case has been presented as a poster at the British Association of Dermatologists 101st Annual Meeting 2021 and a poster abstract has been published in Volume 185, Issue S1, Special Issue: Abstracts for the British Association of Dermatologists 101st Annual Meeting, Virtual Meeting in July 2021 by one of the co-authors (4th author) in this case report, Darren Roche. The title of the poster abstract is; P12: Treatment of primary cutaneous mucormycosis due to Absidia corymbifera in a patient with systemic lupus erythematosus and lupus nephritis. DOI: 10.13140/RG.2.2.28230.80968. I would like to confirm no conflict of interests with Darren Roche, in proceeding with this submission as a consequence of the above poster abstract submission.

\section{References}

1. Ribes JA, Vanover-Sams CL, Baker DJ: Zygomycetes in human disease. Clin Microbiol Rev. 2000, 13:236-301. 10.1128/CMR.13.2.236

2. Nigwekar SU, Thadhani R, Brandenburg VM: Calciphylaxis. N Engl J Med. 2018, 378:1704-1714. 10.1056/NEJMra1505292 